\title{
CD14 Positive Extracellular Vesicles in Broncho-Alveolar Lavage Fluid as a New Biomarker of Acute Respiratory Distress Syndrome
}

\author{
Rahul Y. Mahida ${ }^{1}$, Joshua Price ${ }^{2}$, Sebastian T. Lugg ${ }^{1}$, Hui Li ${ }^{1,3}$, Dhruv Parekh $^{1}$, Aaron Scott $^{1}$, \\ Paul Harrison ${ }^{2}$, Michael A. Matthay ${ }^{4}$, David R. Thickett ${ }^{1}$.
}

${ }^{1}$ Birmingham Acute Care Research Group, Institute of Inflammation and Ageing, University of Birmingham, Birmingham, U.K.

${ }^{2}$ Institute of Inflammation and Ageing, University of Birmingham, Birmingham, U.K.

${ }^{3}$ Department of Anesthesia and Critical Care, The Second Affiliated Hospital and Yuying Children's Hospital of Wenzhou Medical University, Zhejiang, China

${ }^{4}$ Cardiovascular Research Institute, Department of Medicine, and Department of Anesthesia, University of California San Francisco, San Francisco, California, U.S.A.

Corresponding Author: Dr Rahul Y. Mahida

Institute of Inflammation and Ageing, University of Birmingham, Birmingham, B15 2TH, U.K.

Email: r.mahida@bham.ac.uk 


\section{ABSTRACT}

Recent studies have indicated that extracellular vesicles (EV) may play a role in the pathogenesis of Acute Respiratory Distress Syndrome (ARDS). EV have been identified as potential biomarkers of disease severity and prognosis in other pulmonary diseases. We sought to characterize the EV phenotype within ARDS patient broncho-alveolar lavage fluid (BAL), and to determine whether BAL EV could be utilized as a potential biomarker in ARDS. EV from the BAL of sepsis patients with ARDS, sepsis patients without ARDS, and postoperative control patients were characterized with regards to size, number and cell of origin. ARDS patients had significantly higher numbers of $\mathrm{CD} 14^{+} / \mathrm{CD} 81^{+}$monocyte-derived BAL EV than sepsis patients without ARDS $(p=0.022) . C D 14^{+} / C D 81^{+}$BAL EV numbers were significantly higher in those ARDS patients who died during the 30 days following ICU admission $(p=0.027)$. Also, across all sepsis patients there was an association between

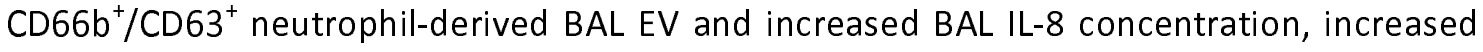
alveolar neutrophil apoptosis and decreased alveolar macrophage efferocytosis. Thus, $\mathrm{CD} 14^{+} / \mathrm{CD} 81^{+} \mathrm{BAL}$ EV are a potential biomarker for disease severity and mortality in ARDS. These findings provide the impetus to further elucidate the contribution of these EV to ARDS pathogenesis.

Key Words: Acute Respiratory Distress Syndrome, Sepsis, Extracellular Vesicles, BronchoAlveolar Lavage, Monocyte. 


\section{INTRODUCTION}

Acute Respiratory Distress Syndrome (ARDS) is a hyper-inflammatory pulmonary disorder, which most commonly develops following sepsis. Neutrophilic inflammation and alveolarcapillary barrier damage lead to alveolar edema and refractory hypoxia, requiring prolonged mechanical ventilation (1). Mortality remains high at 35-45\% (2). Survivors have long-term morbidity and increased risk of developing lung fibrosis. There is an urgent need to identify novel therapeutic targets for ARDS, especially due to the evolving SARS-CoV-2 pandemic in which ARDS is the main cause of mortality (3).

Extracellular vesicles (EV) are membrane-bound anuclear structures which constitute an inter-cellular communication mechanism (4). EV allow targeted transfer of diverse biologic cargo (including mitochondria, RNA, cytokines) between different cell types (5). Uptake of EV can alter gene expression within host cells (6). The tetraspanins CD9, CD63 and CD81 are transmembrane proteins commonly expressed on EV, and can be used to isolate EV in acellular biofluids (7). There are two main subtypes of EV: exosomes and microvesicles. Exosomes are $30-150 \mathrm{~nm}$ in diameter and form by fusion of multi-vesicular bodies with the plasma membrane, whereas microvesicles are $100-1000 \mathrm{~nm}$ in diameter and form by outward blebbing of the plasma membrane (4). With an overlap in size and some common markers (e.g. tetraspanins) differentiating exosomes and microvesicles is challenging, however Endosomal Sorting Complex Required for Transport (ESCRT) proteins such as syntenin are candidate internal cargo marker specific to exosomes (8).

Recent evidence indicates that EV play an important role in ARDS pathogenesis by promoting persistent inflammation. Pathogenic EV are released when human ex-vivo 
medRxiv preprint doi: https://doi.org/10.1101/2021.09.25.21264053; this version posted September 28, 2021. The copyright holder for this

perfused lungs are injured with E. coli; isolation of these EV and administration to uninjured human lungs can also induce inflammatory lung injury (9). Murine models of lipopolysaccharide lung injury have shown that EV transfer of microRNA cargo (e.g. miR-466) to alveolar macrophages can increase inflammatory cytokine release (10). Recent studies have indicated that $\mathrm{CD} 14^{+}$BAL EV may be a potential biomarker for disease activity in COPD (11).

We have previously shown that alveolar macrophages isolated from patients with sepsisrelated ARDS have impaired efferocytosis (ability to clear apoptotic cells) compared to control ventilated sepsis patients without ARDS (12). Alveolar macrophage efferocytosis negatively correlated with alveolar neutrophil apoptosis, and broncho-alveolar lavage (BAL) cytokines IL-8 and IL-1 $\alpha$. Impaired efferocytosis was associated with increased 30-day mortality and duration of mechanical ventilation, indicating that this alveolar macrophage functional defect contributes to ARDS pathogenesis (12). Treatment of healthy alveolar macrophages with BAL from ARDS patients also impairs efferocytosis and downregulates Rac1 expression (which causes cytoskeletal rearrangement allowing apoptotic cell engulfment), reproducing the same functional defect observed in ARDS (13). However, the causative immunomodulatory factors within ARDS patient BAL are yet to be identified. Identification of differentially expressed EV populations within ARDS BAL may guide further investigation into the mechanism of this alveolar macrophage functional defect

In this study, the objective was characterize the EV phenotype of ARDS patient BAL and to compare this EV population to that in control sepsis and post-operative patients at-risk of developing ARDS. We also aimed to determine whether BAL EV could be utilized as a potential biomarker in ARDS. 


\section{MATERIALS AND METHODS}

\section{Ethical Approval}

As previously described (12), ethical approval was obtained to recruit ventilated sepsis patients with and without ARDS (REC 16/WA/0169). Ethical approval was also obtained to recruit patients undergoing transthoracic esophagectomy for esophageal carcinoma (REC $12 / \mathrm{WM} / 0092)$ as previously described in the VINDALOO trial $(14,15)$. For patients who lacked capacity, permission to enroll was sought from a personal legal representative in accordance with the UK Mental Capacity Act (2005). For patients with capacity, written informed consent was obtained from the patient.

\section{Patient recruitment and broncho-alveolar lavage}

Invasively ventilated adult sepsis patients with and without ARDS were recruited from the intensive care unit of the Queen Elizabeth Hospital Birmingham, U.K. from December 2016 February 2019 and BAL was collected as previously described, within 48 hours of initiation of mechanical ventilation (12). Patient demographic and physiological details can also be found in this prior publication. As part of the VINDALOO trial (15), patients underwent postoperative bronchoscopy and BAL following oesophagectomy. Following unblinding of the study, those patients who received placebo and who had not developed ARDS during their post-operative course were identified; BAL samples from 8 of these at-risk post-operative patients were analyzed in this study. For all patients, BAL fluid was rendered acellular by 
medRxiv preprint doi: https://doi.org/10.1101/2021.09.25.21264053; this version posted September 28, 2021. The copyright holder for this

centrifuging at $500 \mathrm{~g}$ for 5 minutes then stored at $-80^{\circ} \mathrm{C}$ prior to use in this study. Differential cell count was performed on cells isolated from BAL.

\section{BAL Extracellular Vesicle characterization}

A single-particle interferometric reflectance image sensing platform was used (Exoview R100 reader, NanoView Biosciences, USA) to detect and characterize EV size, number and surface marker expression within BAL samples (16). The Exoview platform allows quantification and phenotyping of EV as small as 50nm in diameter (7). EV numbers in BAL were measured using ExoView ${ }^{\mathrm{TM}}$ Tetraspanin kits (NanoView Bioscience, USA) according to manufacturer's instructions. Human BAL samples were diluted with incubation solution at a 1:20 dilution. $35 \mu \mathrm{l}$ of diluted BAL was loaded on the ExoView Tetraspanin chips and incubated for 16 hours in a sealed, humidified 24-well plate at room temperature to allow EV binding. Each chip contained spots printed with mouse anti-human antibodies against the tetraspanins CD63 (clone H5C6), CD81 (clone JS81) and CD9 (clone H19a) which enabled EV capture. Chips also contained mouse $\operatorname{lgG} 1 \kappa$ matching isotype antibody, used as a control for non-specific EV binding. Chips were washed three times with incubation solution; after each wash the plate was shaken at 500rpm (LSE digital microplate shaker, Corning, USA). Chips were stained with a fluorescent antibody cocktail for 1 hour at room temperature in the dark to characterize EV surface markers. Fluorophore-conjugated mouse anti-human antibodies included: CD14-APC (1:100 dilution, clone 61D3, Invitrogen), CD206Phycoerythrin (1:100 dilution, clone 19.2, Invitrogen), EpCam-AlexaFluor488 (1:400 dilution, clone 94C, BioLegend), CD66b-AlexaFluor647 (1:200 dilution, clone G10F5, BD Biosciences), CD31-Phycoerythrin (1:100 dilution, clone WM59, Invitrogen), and CD41-AlexaFluor488 
medRxiv preprint doi: https://doi.org/10.1101/2021.09.25.21264053; this version posted September 28, 2021. The copyright holder for this

(1:400 dilution, clone HIP8, BioLegend). Chips were washed thrice as described above, with a final wash in distilled water. Chips were then imaged using the ExoView R100 reader and nScan2 v2.76 software. Data were analyzed using NanoViewer v2.82, using mouse IgG capture spots as negative control (indicative of non-specific binding) for fluorescent gating, and EV sizing thresholds set at 50-200nm diameter. EV cell origin data were correlated with previously recorded clinical outcomes and immune cell functional parameters (12) to identify potential biomarkers. Data from each tetraspanin capture spot (CD63, CD81 and CD9) was calculated separately by subtracting negative control values from IgG spots.

\section{Statistical Analysis}

Data were analyzed using Prism 8 software (GraphPad, USA). Parametric data shown as mean and standard deviation. Non-parametric data shown as median and interquartile range. Differences between continuously distributed data assessed using t-tests for parametric data, or Mann-Whitney tests for non-parametric data. Differences between three or more unpaired non-parametric data sets assessed using Kruskal-Wallis Analysis of Variance (ANOVA) followed by Dunn's multiple comparison tests. Two-tailed p-values of $\leq 0.05$ were considered significant.

\section{RESULTS}

The demographic and clinical characteristics of patients from each of the three cohorts analyzed are shown in Table 1. BAL samples were analyzed from 17 sepsis patients with ARDS, 14 sepsis patients without ARDS and 8 post-operative patients. 
medRxiv preprint doi: https://doi.org/10.1101/2021.09.25.21264053; this version posted September 28, 2021. The copyright holder for this

BAL EV were characterized with regards to size, number and surface marker expression to indicate cellular origin. The size distribution of BAL EV did not differ among the three patient groups (Figure 1A). Representative fluorescence images from the Exoview platform demonstrating EV surface marker expression and lack of non-specific binding are shown in Figure 1B-E.

Characterization studies showed that a greater number of $\mathrm{CD} 14^{+} \mathrm{EV}$ were present in the $\mathrm{BAL}$ of ARDS patients compared to sepsis patients without ARDS, detected by $C D 81^{+}$tetraspanin capture (Figure $2 \mathrm{~A}$, medians $1.23 \times 10^{8} / \mathrm{ml}$ vs $6.26 \times 10^{7} / \mathrm{ml}, \mathrm{p}=0.026$ ). CD66 $\mathrm{b}^{+}$BAL EV were elevated in ARDS patients compared to post-operative control patients, detected by $\mathrm{CD}^{+} 3^{+}$ tetraspanin capture (Figure 2B, medians $4.65 \times 10^{7} / \mathrm{ml}$ vs $5.22 \times 10^{6} / \mathrm{ml}, \mathrm{p}=0.029$ ). $\mathrm{CD} 31^{+}$BAL EV were elevated in post-operative control patients compared to sepsis patients with and without ARDS, detected by $\mathrm{CD}^{+}$tetraspanin capture (Figure $2 \mathrm{C}, \mathrm{p}<0.018$ ). $\mathrm{CD} 41^{+} \mathrm{BAL} \mathrm{EV}$ were decreased in the post-operative control group compared to sepsis patients with and without ARDS, detected by $\mathrm{CD} 3^{+}$tetraspanin capture (Figure 2D, $\mathrm{p}<0.014$ ). There were no differences in the numbers of $\mathrm{CD}_{20}{ }^{+}$and $\mathrm{EpCam}^{+} \mathrm{BAL} \mathrm{EV}$ between sepsis patients with and without ARDS (Figure 2E-F).

In sepsis patients with ARDS, those who died within 30 days of ICU admission had a greater number of $\mathrm{CD} 14^{+} / \mathrm{CD} 81^{+} \mathrm{BAL}$ EV than survivors (Figure $3 \mathrm{~A}$, medians $3.43 \times 10^{8} / \mathrm{ml}$ vs 9.54 $\left.\mathrm{x} 10^{7} / \mathrm{ml}, \mathrm{p}=0.027\right)$, however there is overlap between groups. Across all sepsis patients with and without ARDS, $\mathrm{CD}_{6} 6 \mathrm{~b}^{+} / \mathrm{CD} 3^{+} \mathrm{BAL}$ EV correlated directly with BAL interleukin (IL)-8 concentrations, (Figure 3B, $r=0.751, p<0.0001$ ). Across all sepsis patients with and without ARDS, $\mathrm{CD}_{66 \mathrm{~b}^{+}} / \mathrm{CD} 3^{+} \mathrm{BAL} \mathrm{EV}$ correlated inversely with alveolar macrophage efferocytosis index (Figure 3C, $r=-0.612, p=0.0025$ ). 


\section{DISCUSSION}

In this study, sepsis patients with ARDS had significantly higher numbers of $\mathrm{CD} 14^{+} / \mathrm{CD} 81^{+}$ BAL EV than sepsis patients without ARDS. CD $14^{+} / \mathrm{CD} 81^{+}$BAL EV numbers were significantly higher in those ARDS patients who died during the 30 days following ICU admission. CD $206^{+}$ EV numbers were unchanged in ARDS patients (CD206 being an alveolar macrophage marker), indicating that the increased $\mathrm{CD} 14^{+} \mathrm{EV}$ observed in ARDS patients were derived from infiltrating monocytes as opposed to resident alveolar macrophages. CD14 has previously been used as a marker of monocyte-derived EV (17). The relationship between $\mathrm{CD} 14^{+} / \mathrm{CD} 81^{+} \mathrm{BAL} \mathrm{EV}$ and mortality in ARDS patients is in keeping with previous studies showing that the degree of monocyte influx in ARDS can correlate with the severity of respiratory failure (18). Previous studies have shown that monocyte-derived plasma EV containing gasdermin $\mathrm{D}$ and activated caspase- 1 are more abundant in patients with sepsisrelated ARDS compared to healthy controls; uptake of these EV can induce death in human pulmonary vascular endothelial cells (19). This data supports our hypothesis that $\mathrm{CD}_{14}{ }^{+} \mathrm{EV}$ may contribute to ARDS pathogenesis, and might be utilized as a biomarker of disease severity.

We also observed associations between $\mathrm{CD} 66 \mathrm{~b}^{+} / \mathrm{CD} 3^{+}$neutrophil-derived $\mathrm{BAL} \mathrm{EV}$ in sepsis patients and increased BAL IL-8 concentration and decreased alveolar macrophage efferocytosis. These data indicate that $\mathrm{CD} 6 \mathrm{~b}^{+} / \mathrm{CD} 63^{+} \mathrm{BAL} \mathrm{EV}$ may be a marker of inflammation in ARDS. Neutrophil-derived EV have been shown to have an antiinflammatory, tolerogenic impact following uptake by macrophages and T lymphocytes (2022). Previous studies have also reported that neutrophil-derived EV can transfer microRNA- 
medRxiv preprint doi: https://doi.org/10.1101/2021.09.25.21264053; this version posted September 28, 2021. The copyright holder for this

223 to alveolar epithelial cells, which reduces protein permeability and cytokine release via repression of poly[adenosine diphosphate-ribose] polymerase-1 (PARP-1) (23). Therefore, the increased number of $\mathrm{CD}_{6} 6 \mathrm{~b}^{+} \mathrm{EV}$ in patients with a more inflammatory alveolar microenvironment may be a compensatory response to suppress inflammation and reduce alveolar epithelial injury.

Our findings of decreased $\mathrm{CD} 31^{+} / \mathrm{CD}^{+} \mathrm{EV}$ and increased $\mathrm{CD} 41^{+} / \mathrm{CD} 63^{+} \mathrm{EV}$ in sepsis patients with and without ARDS compared to post-operative control patients may be indicative of pathological processes common to all sepsis patients. Previous studies have shown that platelet-derived EV can induce endothelial cell apoptosis in sepsis patients (24). However, no difference in $\mathrm{CD} 31^{+}$and $\mathrm{CD} 41^{+} \mathrm{EV}$ s was observed between sepsis patients with and without ARDS, therefore conclusions cannot be drawn regarding the relevance of these EV in ARDS pathogenesis.

EV transfer of microRNA cargo to murine macrophages can alter metabolic profile and function (25); EV transfer of long non-coding RNA can promote glycolysis (26). An association between impaired alveolar macrophage efferocytosis and dependence on glycolysis has been observed in COPD patients (27). Further studies are required to determine if similar pathological processes occurs in ARDS, specifically whether $\mathrm{CD} 14^{+} \mathrm{EV}$ transfer of microRNA or protein cargo induces alveolar macrophage dysfunction (12), thereby promoting persistent inflammation. Understanding the role of EV in ARDS pathogenesis will allow identification of potential EV / microRNA biomarkers and novel therapeutic targets for ARDS.

A limitation of our study is that while we identified EV from the major cell types present in the alveolar space, not all cellular origins were included in our analysis (e.g. 
medRxiv preprint doi: https://doi.org/10.1101/2021.09.25.21264053; this version posted September 28, 2021. The copyright holder for this

dendritic cells, lymphocytes). Although tetraspanin capture of EVs provides greater EV specificity compared to other isolation methods, it also limits phenotyping, and therefore only provides an indication of the relevance of $\mathrm{CD} 14^{+} \mathrm{EV}$. The finding that $\mathrm{CD} 14$ most commonly co-localises with tetraspanin CD81, and CD66b with tetraspanin CD63, may indicate different EV biogenesis pathways in monocytes and neutrophils respectively (28). Future studies are required to specifically capture $\mathrm{CD} 14^{+}$and $\mathrm{CD} 66 \mathrm{~b}^{+} \mathrm{EV}$, then to probe external markers for extensive phenotyping, and internal markers (e.g. syntenin) to determine EV biogenesis pathways .

In conclusion, we report that $\mathrm{CD} 14^{+} / \mathrm{CD} 81^{+} \mathrm{BAL} \mathrm{EV}$ are enriched in patients with sepsis-related ARDS compared to controls, and that an elevated $\mathrm{CD} 14^{+} / \mathrm{CD} 81^{+} \mathrm{BAL}$ EV count is associated with increased mortality in ARDS patients, although the sample size is modest. Thus, CD14 ${ }^{+}$BAL EV are a potential biomarker for disease severity and mortality in ARDS. Our findings provide the impetus to further elucidate the role these EV play in ARDS pathogenesis.

\section{Ethical Approval}

Ethical approval was given by the Wales Research Ethics Committee 1 (reference number 16/WA/0169) to recruit ventilated sepsis patients with and without ARDS. Ethical approval was also given by the South Birmingham (England) Research Ethics Committee (reference number 12/WM/0092) to recruit patients undergoing transthoracic esophagectomy for esophageal carcinoma as previously described in the VINDALOO trial. For patients who lacked capacity, permission to enroll was sought from a personal legal representative in accordance with the UK Mental Capacity Act (2005). For patients with capacity, written informed consent was obtained from the patient.

\section{Grants}


medRxiv preprint doi: https://doi.org/10.1101/2021.09.25.21264053; this version posted September 28, 2021. The copyright holder for this preprint (which was not certified by peer review) is the author/funder, who has granted medRxiv a license to display the preprint in perpetuity. All rights reserved. No reuse allowed without permission.

This work was funded by Medical Research Council grants MR/N021185/1 (RYM) and MR/L002736/1 (DT/AS), and by National Heart, Lung and Blood Institute grants HL134828 (MAM) and HL140026 (MAM). The ExoView platform was funded by an EPSRC Capital award awarded to Dr. Sophie Cox, University of Birmingham.

\section{Disclosures}

No conflicts of interest, financial or otherwise, are declared by the authors.

\section{Author Contributions}

RYM, AS, DP, PH, MAM, and DRT contributed to study conception and design. RYM, JP, HL and STL contributed to data acquisition. JP and PH provided assistance and training for use of the Exoview platform. All authors contributed to the data analysis and interpretation. RYM, JP, and DRT drafted the manuscript. All authors critically revised the manuscript for intellectual content and approved the final version before submission.

\section{Figure 1: BAL EV size distribution and representative Exoview fluorescence images}

A) BAL EV size distribution measured using single-particle interferometric reflectance image sensing (Exoview R100 platform). Statistical analysis by 2-way ANOVA, $n \geq 8$ in each group. All data expressed as mean and standard deviation. There was no significant difference in EV size distribution between sepsis patients with ARDS, sepsis patients without ARDS, and postoperative patients ( $p>0.994)$. EV measured between $150-200 \mathrm{~nm}$ are not shown in this graph as these accounted for $<1 \%$ of total EV population in all three groups. Panels B-D show representative Exoview fluorescence images demonstrating EV surface marker expression. B: Detection of $\mathrm{CD}_{66 \mathrm{~b}^{+}} \mathrm{EV}$ (AlexaFluor647) on CD63 tetraspanin spot. C: Detection of $\mathrm{EpCam}^{+} \mathrm{EV}$ (AlexaFluor488) on CD9 tetraspanin spot. D: Detection of $\mathrm{CD}^{+} 1^{+} \mathrm{EV}$ (Phycoerythrin) on CD9 tetraspanin spot. E: Representative Mouse IgG spot negative control showing low non-specific EV binding in BAL samples.

Figure 2: BAL EV surface marker expression in ARDS, sepsis control and post-operative control patients

BAL EV surface marker expression measured by tetraspanin antibody capture (CD9/63/81) and fluorescent antibody labelling via the Exoview R100 platform (EpCam for epithelial, CD66b for neutrophil, CD206 for alveolar macrophage, CD31 for endothelial, CD41 for platelet and CD14 for monocyte origin). All data expressed as median and inter-quartile range, $n \geq 8$ in each group. Statistical analysis by Kruskal-Wallis ANOVA and Dunn's multiple comparisons test. Fluorescence data is presented by individual tetraspanin capture spots. A) 
BAL CD $14^{+}$EV were elevated in ARDS patients compared to sepsis patients without ARDS, detected by $\mathrm{CD} 81^{+}$tetraspanin capture (medians $1.23 \times 10^{8} / \mathrm{ml}$ vs $6.26 \times 10^{7} / \mathrm{ml}, \mathrm{p}=0.026$ ). B) $\mathrm{BAL} C D 66 \mathrm{~b}^{+} \mathrm{EV}$ were elevated in ARDS patients compared to post-operative control patients, detected by $\mathrm{CD}^{+} 3^{+}$tetraspanin capture (medians $4.65 \times 10^{7} / \mathrm{ml}$ vs $5.22 \times 10^{6} / \mathrm{ml}$, $\mathrm{p}=0.029$ ). C) $\mathrm{BAL} C D 31^{+} \mathrm{EV}$ numbers were elevated in post-operative control patients compared to sepsis patients with and without ARDS, detected by $\mathrm{CD}^{+}$tetraspanin capture $(p<0.018)$. D) BAL CD41 ${ }^{+} \mathrm{EV}$ numbers were decreased in the post-operative control group compared to sepsis patients with and without ARDS, detected by $\mathrm{CD}^{+} 3^{+}$tetraspanin capture $(p<0.014)$. E-F) There were no differences in the numbers of $C D 206^{+}$and $E p C a m^{+} E V$ between sepsis patients with and without ARDS.

Figure 3: Correlations between BAL EV and clinical, biochemical and immune parameters AM= Alveolar Macrophage. IL= Interleukin. A) In sepsis patients with ARDS, those who died within 30 days of ICU admission had a greater number of $\mathrm{CD} 14^{+} / \mathrm{CD} 81^{+}$BAL EV than survivors (medians $3.43 \times 10^{8} / \mathrm{ml}$ vs $9.54 \times 10^{7} / \mathrm{ml}, \mathrm{p}=0.027, \mathrm{n}=17$ ). Statistical analysis by Mann-Whitney test, data shown as median and inter-quartile range. B) Across all sepsis patients, with and without ARDS, $\mathrm{CD}_{6} 6 \mathrm{~b}^{+} / \mathrm{CD}^{+} 3^{+}$BAL EV correlate directly with BAL IL-8 concentrations $(r=0.751, p<0.0001, n=31)$. Statistical analyses by Spearman's correlation coefficient. C) Across all sepsis patients, with and without ARDS, CD66 $\mathrm{b}^{+} / \mathrm{CD} 63^{+} \mathrm{BAL} \mathrm{EV}$ correlate inversely with AM efferocytosis index $(r=-0.612, p=0.0025, n=31)$. Statistical analyses by Spearman's correlation coefficient.

\section{REFERENCES}

1. Matthay MA, Zemans RL, Zimmerman GA, Arabi YM, Beitler JR, Mercat A, Herridge M, Randolph AG, and Calfee CS. Acute respiratory distress syndrome. Nature reviews Disease primers 5: 18, 2019.

2. Bellani G, Laffey JG, Pham T, Fan E, Brochard L, Esteban A, Gattinoni L, van Haren F, Larsson A, McAuley DF, Ranieri M, Rubenfeld G, Thompson BT, Wrigge H, Slutsky AS, and Pesenti A. Epidemiology, Patterns of Care, and Mortality for Patients With Acute Respiratory Distress Syndrome in Intensive Care Units in 50 Countries. Jama 315: 788-800, 2016.

3. Yang X, Yu Y, Xu J, Shu H, Xia J, Liu H, Wu Y, Zhang L, Yu Z, Fang M, Yu T, Wang Y, Pan S, Zou X, Yuan S, and Shang Y. Clinical course and outcomes of critically ill patients with SARS-CoV-2 pneumonia in Wuhan, China: a single-centered, retrospective, observational study. The Lancet Respiratory medicine 8: 475-481, 2020.

4. Mahida RY, Matsumoto S, and Matthay MA. Extracellular Vesicles: A New Frontier for Research in Acute Respiratory Distress Syndrome. American journal of respiratory cell and molecular biology 63: 15-24, 2020.

5. Shah R, Patel T, and Freedman JE. Circulating Extracellular Vesicles in Human Disease. The New England journal of medicine 379: 958-966, 2018.

6. Gupta R, Radicioni G, Abdelwahab S, Dang H, Carpenter J, Chua M, Mieczkowski PA, Sheridan JT, Randell SH, and Kesimer M. Intercellular Communication between Airway Epithelial 
Cells Is Mediated by Exosome-Like Vesicles. American journal of respiratory cell and molecular biology 60: 209-220, 2019.

7. $\quad$ Khan NZ, Cao T, He J, Ritzel RM, Li Y, Henry RJ, Colson C, Stoica BA, Faden Al, and Wu J. Spinal cord injury alters microRNA and CD81+ exosome levels in plasma extracellular nanoparticles with neuroinflammatory potential. Brain, behavior, and immunity 92: 165-183, 2021.

8. Kugeratski FG, Hodge K, Lilla S, McAndrews KM, Zhou X, Hwang RF, Zanivan S, and Kalluri R. Quantitative proteomics identifies the core proteome of exosomes with syntenin-1 as the highest abundant protein and a putative universal biomarker. Nature Cell Biology 23: 631-641, 2021.

9. Liu A, Park JH, Zhang X, Sugita S, Naito Y, Lee JH, Kato H, Hao Q, Matthay MA, and Lee JW. Therapeutic Effects of Hyaluronic Acid in Bacterial Pneumonia in the Ex Vivo Perfused Human Lungs. American journal of respiratory and critical care medicine 2019.

10. Shikano S, Gon Y, Maruoka S, Shimizu T, Kozu Y, lida Y, Hikichi M, Takahashi M, Okamoto S, Tsuya K, Fukuda A, Mizumura K, and Hashimoto S. Increased extracellular vesicle miRNA-466 family in the bronchoalveolar lavage fluid as a precipitating factor of ARDS. BMC pulmonary medicine 19: 110, 2019.

11. Bazzan E, Radu CM, Tinè M, Neri T, Biondini D, Semenzato U, Casara A, Balestro E, Simioni $P$, Celi A, Cosio MG, and Saetta M. Microvesicles in bronchoalveolar lavage as a potential biomarker of COPD. American journal of physiology Lung cellular and molecular physiology 320: L241-I245, 2021.

12. Mahida RY, Scott A, Parekh D, Lugg ST, Hardy RS, Lavery GG, Matthay MA, Naidu B, Perkins GD, and Thickett DR. Acute Respiratory Distress Syndrome is associated with impaired alveolar macrophage efferocytosis. The European respiratory journal 2021.

13. Mahida RY, Scott A, Parekh D, Lugg ST, Belchamber KB, Hardy RS, Matthay MA, Naidu B, and Thickett DR. Assessment of alveolar macrophage dysfunction using an in vitro model of Acute Respiratory Distress Syndrome. Front Med (Lausanne) In Press., 2021. DOI: 10.3389/fmed.2021.737859

14. Parekh D, Dancer RC, Lax S, Cooper MS, Martineau AR, Fraser WD, Tucker O, Alderson D, Perkins GD, Gao-Smith F, and Thickett DR. Vitamin D to prevent acute lung injury following oesophagectomy (VINDALOO): study protocol for a randomised placebo controlled trial. Trials 14: 100, 2013.

15. Parekh D, Dancer RCA, Scott A, D'Souza VK, Howells PA, Mahida RY, Tang JCY, Cooper MS, Fraser WD, Tan L, Gao F, Martineau AR, Tucker O, Perkins GD, and Thickett DR. Vitamin D to Prevent Lung Injury Following Esophagectomy-A Randomized, Placebo-Controlled Trial. Critical care medicine 46: e1128-e1135, 2018.

16. Price J, Gardiner C, and Harrison P. Platelet-enhanced plasma: Characterization of a novel candidate resuscitation fluid's extracellular vesicle content, clotting parameters, and thrombin generation capacity. Transfusion 61: 2179-2194, 2021.

17. Wang GH, Lu J, Ma KL, Zhang Y, Hu ZB, Chen PP, Lu CC, Zhang XL, and Liu BC. The Release of Monocyte-Derived Tissue Factor-Positive Microparticles Contributes to a Hypercoagulable State in Idiopathic Membranous Nephropathy. Journal of atherosclerosis and thrombosis 26: 538-546, 2019.

18. Rosseau S, Hammerl P, Maus U, Walmrath HD, Schutte H, Grimminger F, Seeger W, and Lohmeyer J. Phenotypic characterization of alveolar monocyte recruitment in acute respiratory distress syndrome. American journal of physiology Lung cellular and molecular physiology 279: L2535, 2000.

19. Mitra S, Exline M, Habyarimana F, Gavrilin MA, Baker PJ, Masters SL, Wewers MD, and Sarkar A. Microparticulate Caspase 1 Regulates Gasdermin D and Pulmonary Vascular Endothelial Cell Injury. American journal of respiratory cell and molecular biology 59: 56-64, 2018.

20. Shen G, Krienke S, Schiller P, Nießen A, Neu S, Eckstein V, Schiller M, Lorenz HM, and Tykocinski LO. Microvesicles released by apoptotic human neutrophils suppress proliferation and IL2/IL-2 receptor expression of resting T helper cells. European journal of immunology 47: 900-910, 2017. 
21. Eken C, Martin PJ, Sadallah S, Treves S, Schaller M, and Schifferli JA. Ectosomes released by polymorphonuclear neutrophils induce a MerTK-dependent anti-inflammatory pathway in macrophages. The Journal of biological chemistry 285: 39914-39921, 2010.

22. Gasser $\mathbf{0}$, and Schifferli JA. Activated polymorphonuclear neutrophils disseminate antiinflammatory microparticles by ectocytosis. Blood 104: 2543-2548, 2004.

23. Neudecker V, Brodsky KS, Clambey ET, Schmidt EP, Packard TA, Davenport B, Standiford TJ, Weng T, Fletcher AA, Barthel L, Masterson JC, Furuta GT, Cai C, Blackburn MR, Ginde AA, Graner MW, Janssen WJ, Zemans RL, Evans CM, Burnham EL, Homann D, Moss M, Kreth S, Zacharowski K, Henson PM, and Eltzschig HK. Neutrophil transfer of miR-223 to lung epithelial cells dampens acute lung injury in mice. Science translational medicine 9: 2017.

24. Gambim MH, de Oliveira do Carmo A, Marti L, Veríssimo-Filho S, Lopes LR, and Janiszewski M. Platelet-derived exosomes induce endothelial cell apoptosis through peroxynitrite generation: experimental evidence for a novel mechanism of septic vascular dysfunction. Critical Care 11: R107, 2007.

25. Park JE, Dutta B, Tse SW, Gupta N, Tan CF, Low JK, Yeoh KW, Kon OL, Tam JP, and Sze SK. Hypoxia-induced tumor exosomes promote M2-like macrophage polarization of infiltrating myeloid cells and microRNA-mediated metabolic shift. Oncogene 38: 5158-5173, 2019.

26. Chen F, Chen J, Yang L, Liu J, Zhang $X$, Zhang $Y$, Tu Q, Yin D, Lin D, Wong PP, Huang D, Xing Y, Zhao J, Li M, Liu Q, Su F, Su S, and Song E. Extracellular vesicle-packaged HIF-1 $\alpha$-stabilizing IncRNA from tumour-associated macrophages regulates aerobic glycolysis of breast cancer cells. Nature cell biology 21: 498-510, 2019.

27. Ryan E, Coelho P, Cole J, Bewley M, Budd R, Callahan J, McCafferty J, Singh D, Dockrell D, Walmsley $S$, and Whyte $M$. T1 Defective metabolism drives macrophage dysfunction in COPD. Thorax 76: A1-A1, 2021.

28. Mathieu M, Névo N, Jouve $M$, Valenzuela JI, Maurin M, Verweij FJ, Palmulli R, Lankar D, Dingli F, Loew D, Rubinstein E, Boncompain G, Perez F, and Théry C. Specificities of exosome versus small ectosome secretion revealed by live intracellular tracking of CD63 and CD9. Nature communications 12: 4389, 2021. 
Table 1: Patient demographic and clinical characteristics

\begin{tabular}{|c|c|c|c|}
\hline & $\begin{array}{c}\text { Sepsis patients } \\
\text { with ARDS } \\
(n=17)\end{array}$ & $\begin{array}{c}\text { Sepsis patients } \\
\text { without ARDS } \\
(n=14)\end{array}$ & $\begin{array}{c}\text { Post-operative } \\
\text { patients } \\
(n=8)\end{array}$ \\
\hline \multicolumn{4}{|l|}{ Demographics } \\
\hline Age in years: & $59.2(13.9)$ & $55.1(16.3)$ & $65.8(7.9)$ \\
\hline Male Gender: $\mathbf{n}(\%)$ & $15(71 \%)$ & $11(65 \%)$ & $4(50 \%)$ \\
\hline Ethnicity: Caucasian - n (\%) & $21(100 \%)$ & $17(100 \%)$ & $7(88 \%)$ \\
\hline \multicolumn{4}{|l|}{ Smoking status: $\mathbf{n}(\%)$} \\
\hline - Current & $7(33 \%)$ & $5(29 \%)$ & $0(0 \%)$ \\
\hline - Ex-smoker & $9(43 \%)$ & $6(35 \%)$ & $6(75 \%)$ \\
\hline - $\quad$ Never smoker & $2(10 \%)$ & $1(6 \%)$ & $2(25 \%)$ \\
\hline - Unknown & $3(14 \%)$ & $5(29 \%)$ & $0(0 \%)$ \\
\hline $\begin{array}{l}\text { ICU LoS in days: } \\
\text { median (IQR) }\end{array}$ & $\begin{array}{c}23.0 \\
(12.8-33.8)\end{array}$ & $\begin{array}{c}12.0 \\
(7.5-19.0)\end{array}$ & $\begin{array}{c}4.5 \\
(2.5-7.8)\end{array}$ \\
\hline $\begin{array}{l}\text { Hospital LoS in days: } \\
\text { median (IQR) }\end{array}$ & $\begin{array}{c}33.0 \\
(15.3-52.5)\end{array}$ & $\begin{array}{c}24.0 \\
(13.0-39.5)\end{array}$ & $\begin{array}{c}12 \\
(8.3-15.8)\end{array}$ \\
\hline Ventilator Free Days to day 28: & $6.9(9.2)$ & $15.9(8.3)$ & $26.3(1.8)$ \\
\hline Inpatient Mortality: $\mathbf{n}(\%)$ & $7(33.3 \%)$ & $3(17.6 \%)$ & $0(0 \%)$ \\
\hline $\begin{array}{l}\text { BAL leukocyte count } \times 10^{6} \text { : } \\
\text { median (IQR) }\end{array}$ & $\begin{array}{c}15.8 \\
(7.4-31.3)\end{array}$ & $\begin{array}{c}6.4 \\
(3.8-27.0)\end{array}$ & $\begin{array}{c}7.8 \\
(2.1-10.5)\end{array}$ \\
\hline \% Neutrophils in BAL: & $69.9(21.5)$ & $49.0(30.6)$ & $9.5(10.1)$ \\
\hline
\end{tabular}

$I Q R=$ Interquartile range. $L O S=$ Length of stay. ICU = Intensive care unit. Data are presented as mean (standard deviation) unless otherwise indicated. 


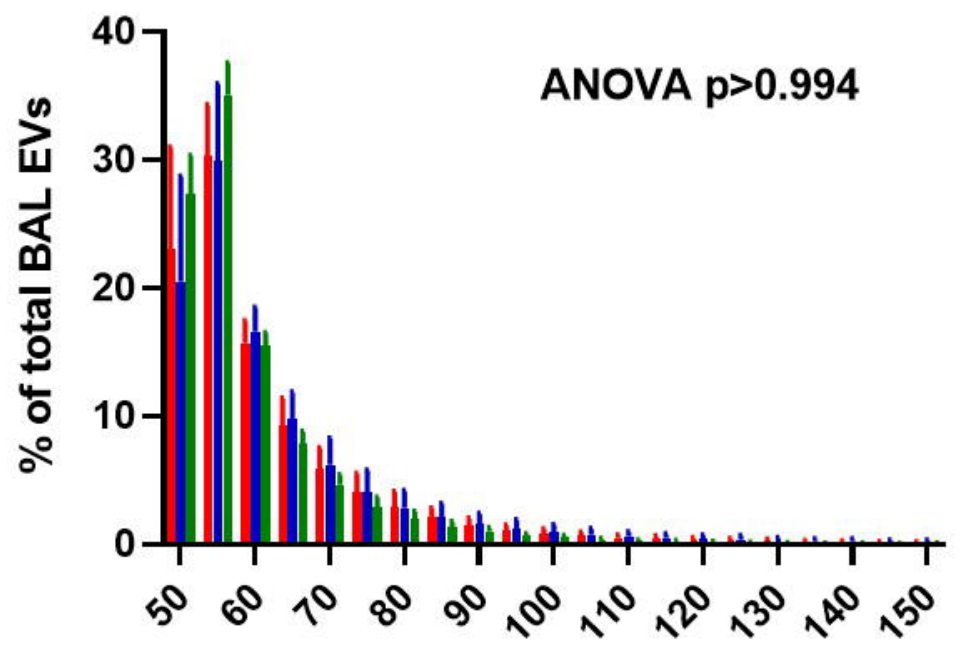

- Sepsis with ARDS $(n=17)$

- Sepsis without ARDS ( $n=14)$

- Post-operative Controls $(n=8)$

A EV Diameter $(\mathrm{nm})$

B

C

D

E 


\section{CD9+}

A

CD14+

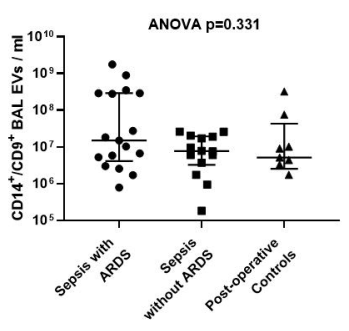

B CD66b+

C

CD31+

D

CD41+

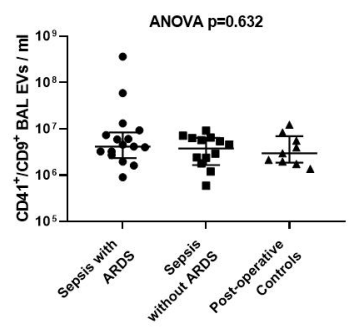

E

EpCam+

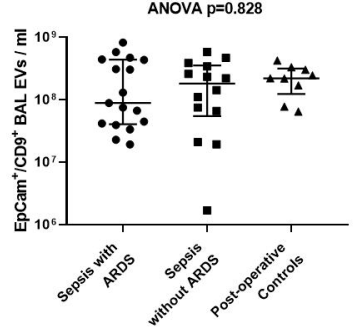

F

CD206+

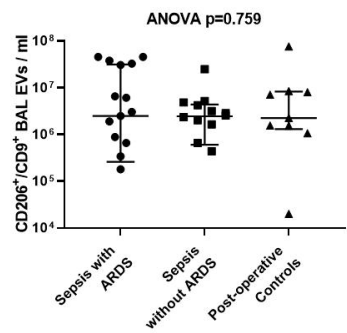

CD63+

CD81+
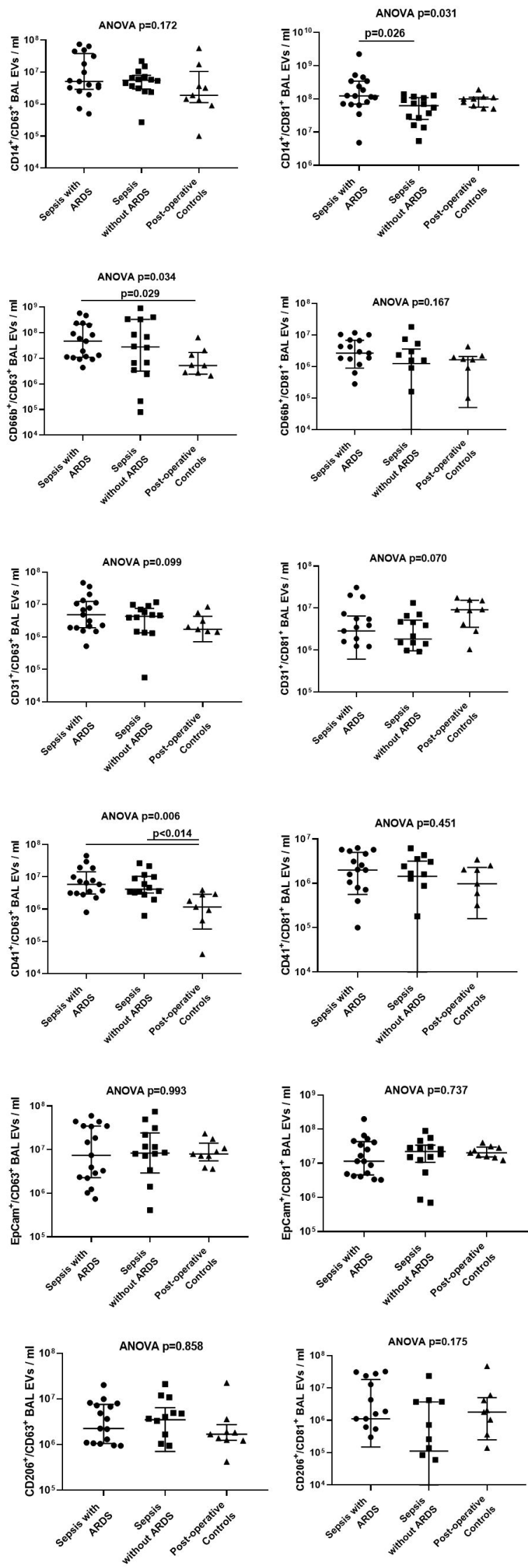
ARDS Patients Only

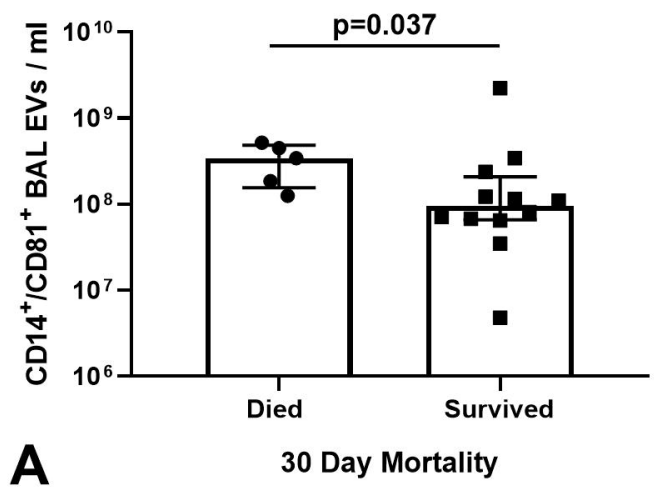

All Sepsis Patients

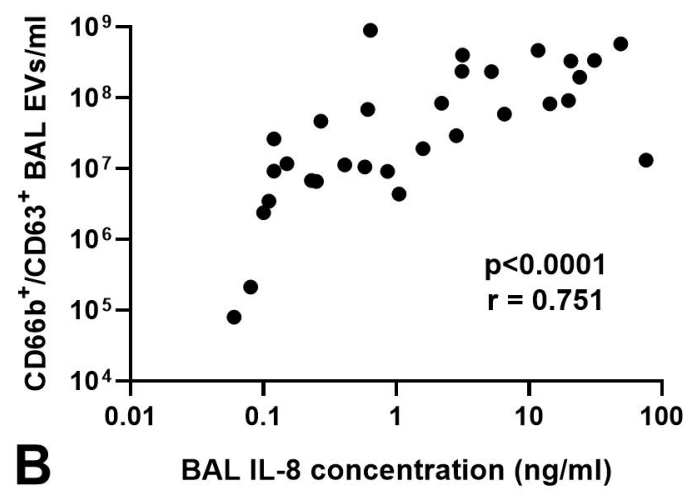

All Sepsis Patients

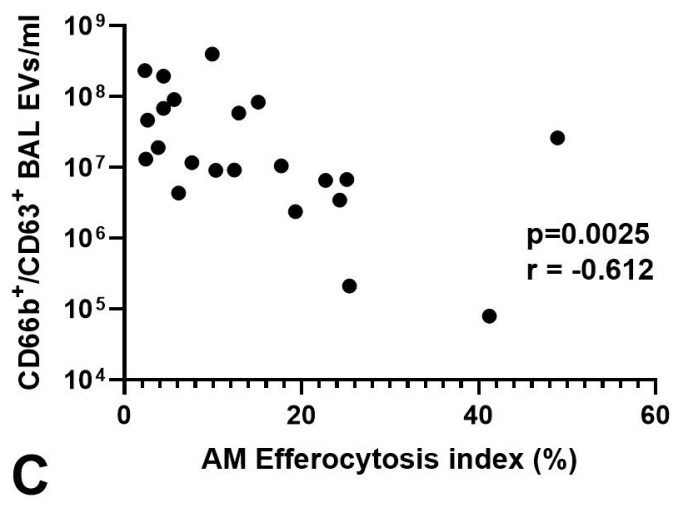

